\title{
Antenna Subset Selection for MIMO Channels With Mutual Coupling
}

\author{
Dithanon Khrutmuang and Wiroonsak Santipach \\ Department of Electrical Engineering \\ Faculty of Engineering, Kasetsart University \\ Bangkok, Thailand 10900 \\ Email: dithanon@hotmail.com and wiroonsak.s@ku.ac.th
}

\begin{abstract}
In this work, we propose antenna selection schemes for a point-to-point multiantenna channel. The schemes select subsets of either transmit and/or receive antennas to activate to increase ergodic capacity. The channel model considered includes spatial correlation and mutual coupling among adjacent antennas, which can have significant impact on the channel performance. Our proposed schemes are shown to perform close to the optimal exhaustive search with less computational complexity.
\end{abstract}

\section{INTRODUCTION}

A capacity of a multiple-input and multiple-output (MIMO) wireless channel has been shown to increase linearly with the minimum number of transmit and receive antennas [1], [2]. However, the linear capacity growth is applied only to a fading channel model with ideal scattering and no mutual coupling among transmitting or receiving antennas. If the scattering in the environment is not sufficiently rich, then there exists spatial correlation among channel gains between transmit and receive antenna pair. Mutual coupling is an electromagnetic phenomenon, which intrinsically exists in an antenna array, and its effect is detrimental to the capacity when antenna is placed sufficiently close to one another [3], [4]. These practical factors could affect the performance of a MIMO channel significantly and should be taken into account in any reasonable channel model.

To increase the performance of multiantenna link, we propose antenna selection schemes that incorporate mutual coupling consideration. Selecting subset of antennas has been shown to increase channel capacity. Furthermore, using only the selected set of antennas also reduces hardware complexity, e.g. , a number of installed radio-frequency (RF) chains needed on either ends of communication link. Antenna selection has been long considered in the literature [5]-[10]. References [6][9] proposed fast suboptimal selection algorithms, which perform close to the exhaustive search over all possible antenna subsets. Assuming MIMO channel with spatial correlations between antennas, [10] proposed the scheme to select receive antennas based on correlations.

All of the work previously mentioned do not consider mutual coupling among antennas, which is shown to greatly affect capacity when antennas are placed very close to one

This work was supported by Kasetsart University Research and Development Institute (KURDI) under the FY2012 Kasetsart University research grant. another [3], [4]. In this work, we propose suboptimal antenna selection schemes for MIMO channel model with both spatial correlation and mutual coupling. The objective of the proposed schemes is to maximize the capacity over potential antenna subsets whose mutual impedance is not to exceed a given threshold. From numerical examples shown, our schemes perform close to the exhaustive search, but require less computation time.

\section{Channel Model}

We consider a point-to-point MIMO channel with $N_{t}$ transmit and $N_{r}$ receive antennas. For a discrete-time channel model, the $N_{r} \times 1$ received vector is given by

$$
\boldsymbol{y}=\boldsymbol{H} \boldsymbol{x}+\boldsymbol{n}
$$

where $\boldsymbol{H}$ is an $N_{r} \times N_{t}$ channel matrix whose element $h_{i, j}$ is the channel gain between the $j$ th transmit and the $i$ th receive antennas, $\boldsymbol{x}$ is an $N_{t} \times 1$ transmitted vector with zero mean and covariance $I$, which is the identity matrix. The transmitted signal is corrupted by additive white Gaussian noise (AWGN) vector $\boldsymbol{n}$, whose mean and covariance are zero and $\sigma_{n}^{2} \boldsymbol{I}$, respectively.

Channel gain $h_{i, j}$ depends on channel model, antenna type, and antenna-array arrangement. Placing antennas close to one another induces a mutual coupling between them. Taking into account both effects of spatial correlation and mutual coupling, the channel matrix is given by

$$
\boldsymbol{H}=\frac{1}{c_{t} c_{r}} \boldsymbol{C}_{r} \boldsymbol{R}^{\frac{1}{2}} \boldsymbol{H}_{w} \boldsymbol{S}^{\frac{1}{2}} \boldsymbol{C}_{t}
$$

where, for an ideal Rayleigh fading channel, $\boldsymbol{H}_{w}$ is an $N_{r} \times N_{t}$ matrix of independent zero-mean unit-variance complex Gaussian random variables, $\boldsymbol{S}$ is the $N_{t} \times N_{t}$ transmit correlation matrix, $\boldsymbol{R}$ is the $N_{r} \times N_{r}$ receive correlation matrix, the $N_{t} \times N_{t}$ matrix $C_{t}$ and $N_{r} \times N_{r}$ matrix $C_{r}$ reflect mutual coupling effect at the transmit-antenna and receive-antenna arrays, respectively, and $c_{t}$ and $c_{r}$ are normalizing constants.

We assume that each antenna has a uniformly distributed angular spread. Thus, from Jakes' model [11], the $(i, j)$ element of the transmit correlation matrix is given by

$$
\boldsymbol{S}_{i, j}=J_{0}\left(\frac{2 \pi d_{i, j}}{\lambda}\right), \quad 1 \leq i, j \leq N_{t}
$$

where $J_{0}(\cdot)$ is the zeroth-order Bessel function, and $d_{i, j}$ is the distance between the $i$ th and $j$ th transmit antennas, and 
$\lambda$ is the transmission wavelength. We assume that antennas are placed uniformly and the length of the antenna array is $L$. Hence,

$$
d_{i, j}=\frac{L|i-j|}{N_{t}-1}
$$

The receive correlation matrix $\boldsymbol{R}$ is similarly defined as the transmit correlation matrix $\boldsymbol{S}$ in (3).

According to [12], the two matrices reflecting mutual coupling effect at the transmit and receive arrays are given by

$$
\begin{aligned}
& \boldsymbol{C}_{t}=\boldsymbol{Z}_{t}\left(\boldsymbol{Z}_{t}+\boldsymbol{Z}_{s}\right)^{-1} \\
& \boldsymbol{C}_{r}=\left(\boldsymbol{Z}_{r}+\boldsymbol{Z}_{l}\right)^{-1}
\end{aligned}
$$

where $Z_{t}$ is an $N_{t} \times N_{t}$ transmit impedance matrix whose diagonal elements are self impedance and whose off-diagonal elements are mutual impedance, and $\boldsymbol{Z}_{s}$ denotes a diagonal matrix whose diagonal elements are the conjugate of the diagonal entries of $\boldsymbol{Z}_{t}$. For a dipole array, $\boldsymbol{Z}_{t}$ is well known from standard antenna theory (e.g., see [12, Ch. 8]). Similarly, $\boldsymbol{Z}_{r}$ is the $N_{r} \times N_{r}$ receive impedance matrix and $\boldsymbol{Z}_{l}$ is a diagonal matrix whose diagonal entries are the conjugate of the diagonal entries of $\boldsymbol{Z}_{r}$. Finally, the two normalizing constants are defined as follows

$$
\begin{gathered}
c_{t}=\frac{\left(\boldsymbol{Z}_{t}\right)_{11}}{\left(\boldsymbol{Z}_{t}\right)_{11}+\left(\boldsymbol{Z}_{t}\right)_{11}^{*}} \\
c_{r}=\frac{\left(\boldsymbol{Z}_{r}\right)_{11}}{\left(\boldsymbol{Z}_{r}\right)_{11}+\left(\boldsymbol{Z}_{r}\right)_{11}^{*}}
\end{gathered}
$$

where $(\cdot)^{*}$ denotes complex conjugate.

Assuming that the channel matrix is known perfectly at the transmitter and receiver, we would like to maximize the performance of the channel by selectively activating subsets of transmit and/or receive antennas. For a transmit-antenna selection, we let $\boldsymbol{F}_{t}$ denote an $N_{t} \times N_{t}$ diagonal matrix whose $i$ th diagonal entry is 1 if the $i$ th transmit antenna is chosen or 0 , otherwise. Similarly, we let $\boldsymbol{F}_{r}$ denote the $N_{r} \times N_{r}$ receiveantenna selection matrix. Let $L_{t}$ be the number of selected or activated transmit antennas, and $L_{r}$ be the number of selected receive antennas. Thus, after the transmit and receive selections, the effective channel matrix is given by $\boldsymbol{F}_{r} \boldsymbol{H} \boldsymbol{F}_{t}$ and the associated ergodic capacity is given by

$$
C=E\left[\log \operatorname{det}\left(\boldsymbol{I}+\frac{\rho}{L_{t}} \boldsymbol{F}_{r} \boldsymbol{H} \boldsymbol{F}_{t} \boldsymbol{H}^{\dagger} \boldsymbol{F}_{r}\right)\right]
$$

where the expectation is over channel distribution and $\boldsymbol{F}_{t} \boldsymbol{F}_{t}^{\dagger}=$ $\boldsymbol{F}_{t}$. We note that antennas that are not selected still induce coupling with adjacent antennas. However, we assume that this mutual coupling from passive antennas is small and can be discarded. Thus, (9) is also a capacity of the link equipped with selected antennas only and all other antennas removed.

In this work, we propose algorithms to determine $F_{t}$ and/or $F_{r}$ that maximize channel capacity for the channel model with mutual coupling.

\section{SElection Algorithms}

In this section, we describe the proposed algorithms to select a set of transmit antennas, a set of receive antennas, or a set of both transmit and receive antennas. The optimal selection scheme that maximizes the channel capacity employs exhaustive search over all possible subsets of antennas. However, search complexity can be tremendous when the number of antenna is large. Here we propose the selection schemes that take into account mutual coupling, reduce the search complexity, and perform close to the optimum.

\section{A. Selection At Either Transmit or Receive Antenna Arrays}

We start with selection for a single antenna array at either end of a communication link. This selection algorithm is based on that proposed by [9], [13] in which a single antenna is chosen at each step sequentially. To select $L_{t}$ transmit antennas, we assume that all receive antennas are active $\left(L_{r}=N_{r}\right.$ and thus, $\boldsymbol{F}_{r}=\boldsymbol{I}$ ). Let $\boldsymbol{e}_{j}$ be an $N_{t} \times 1$ vector whose $j$ th entry is one and all other entries are zero and $e_{j}$ also indicates selecting the $j$ th antenna. For example, $e_{3}=\left[\begin{array}{llllll}0 & 0 & 1 & 0 & \cdots & 0\end{array}\right]^{T}$. We also let $\Omega_{s}$ and $\Omega_{n}$ be the sets of $e_{j}$ 's that correspond to selected antennas and the remaining antennas, respectively.

The proposed algorithm starts with empty $\Omega_{s}$ and a full $\Omega_{n}=\left\{\boldsymbol{e}_{1}, \ldots, \boldsymbol{e}_{N_{t}}\right\}$. Given channel matrix $\boldsymbol{H}$, we select the single transmit antenna that maximizes an instantaneous capacity. If $j$ th antenna is selected, we move the corresponding $e_{j}$ from $\Omega_{n}$ to $\Omega_{s}$. To select subsequent antennas, we determine first if a ratio between mutual impedance between a potential antenna in $\Omega_{n}$ and all selected antennas in $\Omega_{s}$ and the self impedance exceeds a threshold $\epsilon$, where $0<\epsilon<1$. If the ratio exceeds $\epsilon$, we will not consider that antenna as a candidate. From a set of candidate antennas denoted by $\Omega_{c}$, we again find the antenna that maximizes the instantaneous capacity and add it to $\Omega_{s}$. If $\Omega_{c}$ is empty, we set $\Omega_{c}$ to equal $\Omega_{n}$. We iterate until $L_{t}$ antennas are chosen. Detailed steps are shown in Algorithm 1. We note that $\epsilon$ limits the number of candidate antennas in $\Omega_{c}$. If $\epsilon$ is set too low, $\Omega_{c}$ may contain too few antennas. If $\epsilon$ is set too high, $\Omega_{c}$ will be similar to $\Omega_{u}$. In this work, we obtain $\epsilon$ from numerical experiments.

To select receive antennas, similar steps apply. We set $L_{t}=$ $N_{t}$ and hence, $\boldsymbol{F}_{t}=\boldsymbol{I}$. Similar to the transmit selection in Algorithm 1, receive antennas are selected sequentially based on instantaneous capacity and mutual impedance.

\section{B. Selection At Both Transmit and Receive Antenna Arrays}

We would also like to select both transmit and receive antennas that maximize the capacity. Employing exhaustive search may not possible since the number of possible sets of transmit and receive antennas, which equals $\left(\begin{array}{l}N_{t} \\ L_{t}\end{array}\right)\left(\begin{array}{l}N_{r} \\ L_{r}\end{array}\right)$, could be forbiddingly high. To lessen search complexity, we can apply Algorithm 1 to select first the transmit antennas and then the receive antennas or vice versa. In other words, Algorithm 1 is used twice in sequence and the number of possible capacity computations will be at most $\left(\begin{array}{l}N_{t} \\ L_{t}\end{array}\right)+\left(\begin{array}{l}N_{r} \\ L_{r}\end{array}\right)$. However, the performance consequently suffers as later shown by numerical examples in Section IV.

To improve upon the performance of Algorithm 1, we propose Algorithm 2, which is based on the exhaustive search. However, the number of the possible subsets of antennas is reduced by a constraint on mutual impedance. For either transmit or receive sides, we only consider the set of antennas 


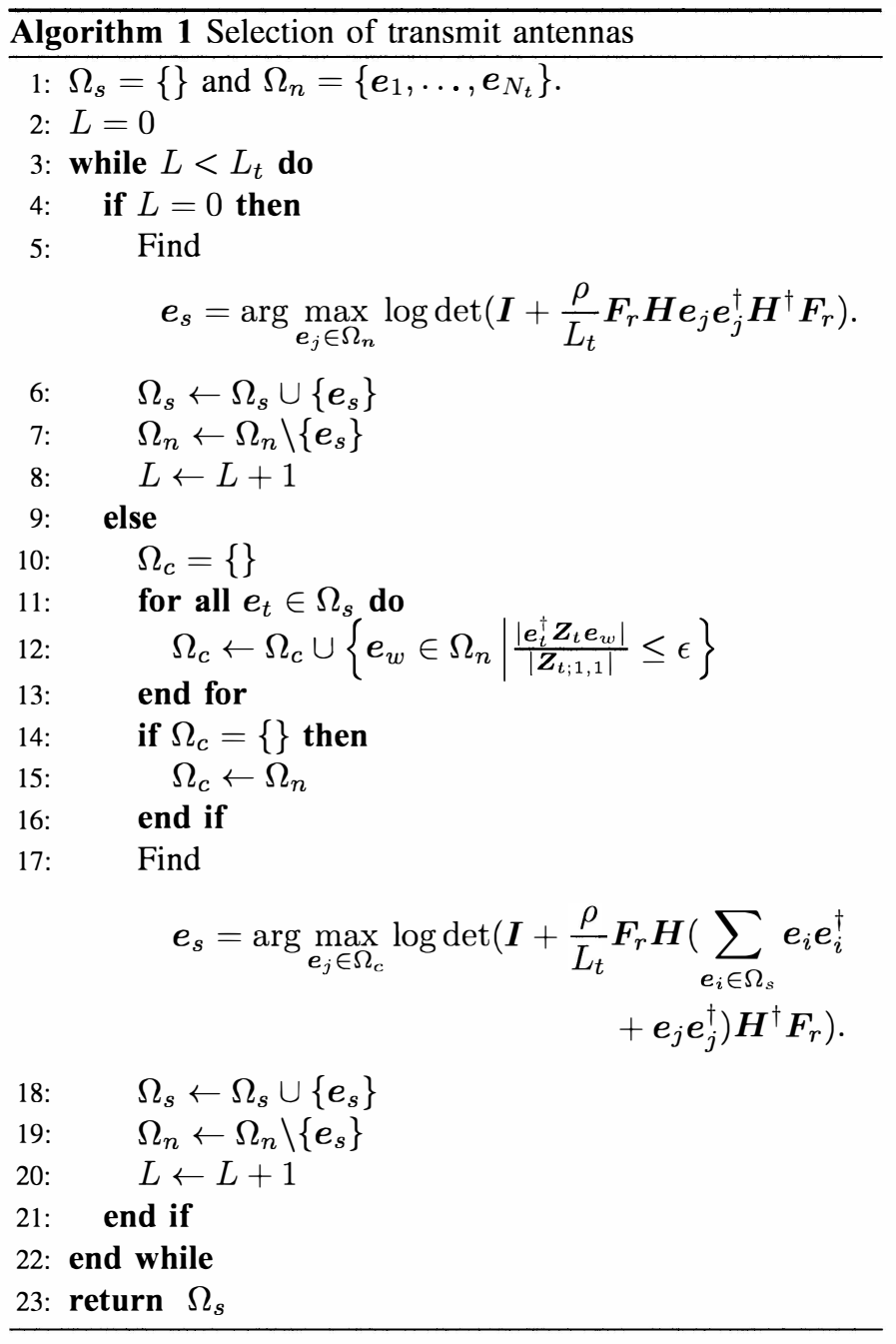

whose ratio between mutual impedance and self impedance does not exceed the threshold $\epsilon$.

Let $\mathcal{F}_{t}=\left\{\boldsymbol{F}_{t}^{(i)}\right\}$, where $1 \leq i \leq\left(\begin{array}{l}N_{t} \\ L_{t}\end{array}\right)$, denote the set of all possible transmit-antenna selection matrices and $\mathcal{F}_{r}=$ $\left\{\boldsymbol{F}_{r}^{(j)}\right\}$, where $1 \leq j \leq\left(\begin{array}{l}N_{r} \\ L_{r}\end{array}\right)$, denote the set of all possible receive-antenna selection matrices. We note that nonzero offdiagonal entries of $\boldsymbol{F}_{t}^{(i)} \boldsymbol{Z}_{t} \boldsymbol{F}_{t}^{(i)}$ are mutual impedance of the transmit antennas associated with $\boldsymbol{F}_{t}^{(i)}$. Similarly, nonzero offdiagonal entries of $\boldsymbol{F}_{r}^{(j)} \boldsymbol{Z}_{r} \boldsymbol{F}_{r}^{(j)}$ are mutual impedance of the receive antennas associated with $\boldsymbol{F}_{r}^{(j)}$. Steps of this proposed algorithm is shown in Algorithm 2.

\section{NumeriCAL RESUlTS}

In all simulation results shown, we assume dipole antenna whose radius is $0.001 \lambda$ and whose length is $0.25 \lambda$. All antennas in the array are uniformly placed side by side. In Fig 1, an ergodic capacity of transmit selection scheme in Algorithm 1 is shown with $N_{t}$ for $\rho=0 \mathrm{~dB}, L_{t}=2$, $N_{r}=L_{r}=3$, and threshold $\epsilon=0.8$. Lengths of both arrays and are set to $L=\lambda$. When $N_{t}$ is large, transmit antennas are placed very close together and hence, induce a large mutual coupling. From the figure, our proposed scheme, exhaustive search, and the scheme proposed by Gorokhov [8] performs
Algorithm 2 Selection of transmit antennas and receive antennas

1: Start with $\mathcal{F}_{t}$ and $\mathcal{F}_{r}$.

2: Find

$$
\begin{aligned}
\overline{\mathcal{F}}_{t}=\left\{\boldsymbol{F}_{t} \in \mathcal{F}_{t} \mid\right. \text { all nonzero } & \text { off-diagonal entries } \\
& \text { of } \left.\boldsymbol{F}_{t} \boldsymbol{Z}_{t} \boldsymbol{F}_{t}<\epsilon \boldsymbol{Z}_{t ; 1,1}\right\} .
\end{aligned}
$$

3: Find

$$
\begin{array}{r}
\overline{\mathcal{F}}_{r}=\left\{\boldsymbol{F}_{r} \in \mathcal{F}_{r} \mid\right. \text { all nonzero off-diagonal entries } \\
\left.\qquad \text { of } \boldsymbol{F}_{r} \boldsymbol{Z}_{r} \boldsymbol{F}_{r}<\epsilon \boldsymbol{Z}_{r ; 1,1}\right\} .
\end{array}
$$

4: Find

$$
\boldsymbol{F}_{t}^{*}, \boldsymbol{F}_{r}^{*}=\arg \max _{\substack{\boldsymbol{F}_{t} \in \overline{\mathcal{F}}_{t} \\ \boldsymbol{F}_{r} \in \overline{\mathcal{F}}_{r}}} \log \operatorname{det}\left(\boldsymbol{I}+\frac{\rho}{L_{t}} \boldsymbol{F}_{r} \boldsymbol{H} \boldsymbol{F}_{t} \boldsymbol{H}^{\dagger} \boldsymbol{F}_{r}\right) .
$$

5: return $\boldsymbol{F}_{t}^{*}$ and $\boldsymbol{F}_{r}^{*}$

similarly. Similar to [13], we modify the Gorokhov scheme by starting with an empty set of selected antennas and adding one antenna at each step to the set. However, based on simulation results not shown here, our scheme is the least complex among the three schemes ${ }^{1}$ We also plot the capacity of selecting all transmit antennas and see a significant performance loss.

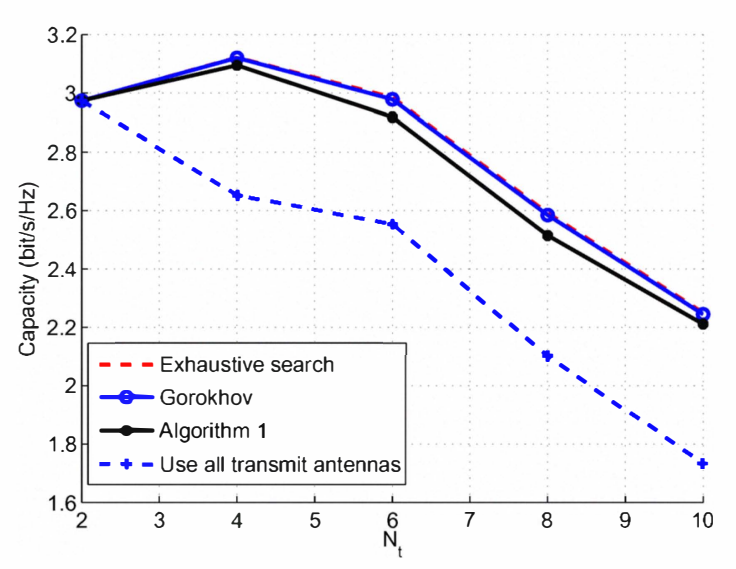

Fig. 1. Capacity with transmit selection scheme in Algorithm 1 is shown with $N_{t}$ for $\rho=0 \mathrm{~dB}, L_{t}=2, N_{r}=L_{r}=3, L=\lambda$, and $\epsilon=0.8$.

With $N_{t}=N_{r}=10$, we would like to find the optimal number of selected transmit and receive antennas. Fig. 2 shows the capacity versus $L_{t}$ or $L_{r}$. For each plot, algorithm 1 was applied to either transmit or receive arrays while all antennas in the other array are active. With transmit selection, the optimal $L_{t}=3$ when $\rho=5 \mathrm{~dB}$ and lengths of both arrays equal $0.5 \lambda$. Comparing to using all transmit antennas, we can gain almost $20 \%$ from activating only subset of antennas. For receiveantenna selection, using all receive antennas gives the capacity maximum. This is due to the fact that more energy is captured by a larger number of receive antennas. However, more RF chains also need to be installed with a larger cost.

\footnotetext{
${ }^{1}$ The associated numerical result is not shown here due to limited space.
} 


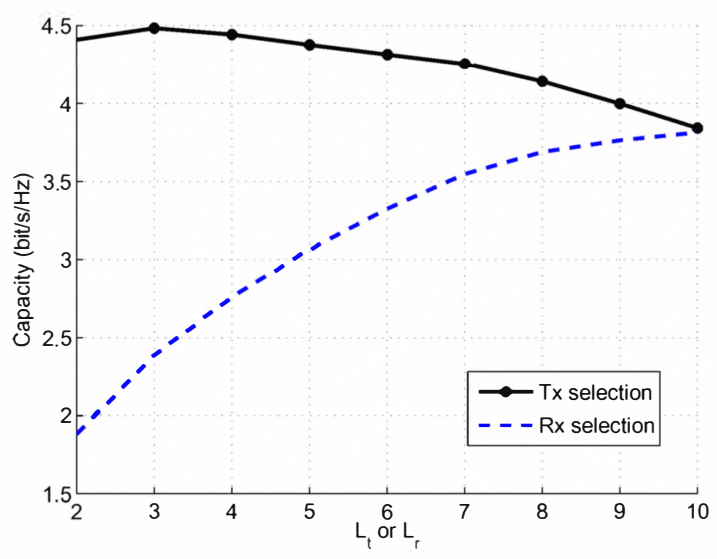

Fig. 2. Capacity with either transmit or receive selection scheme is shown with $L_{t}$ or $L_{r}$ for $\rho=5 \mathrm{~dB}, N_{t}=N_{r}=10, L=0.5 \lambda$, and $\epsilon=0.8$.

In Fig. 3 and 4 , both transmit and receive antennas are selected with $\rho=0 \mathrm{~dB}, L_{t}=L_{r}=3, L=0.5 \lambda$, and $N_{t}=N_{r}$. We note that thresholds for Algorithms 1 and 2 are 0.8 and 0.3 , respectively. We apply Algorithm 2 to find the set of selected transmit and receive antennas, which is then compared to the exhaustive search and also Algorithm 1. All three schemes give similar rate performance with the maximum attained when $N_{t}=N_{r}=5$. Complexity of the three schemes is measured by the computation time and is shown in Fig. 4. We note that computation time of exhaustive search increases exponentially with the number of available antennas while those of our proposed schemes are less, especially computation time of Algorithm 1.

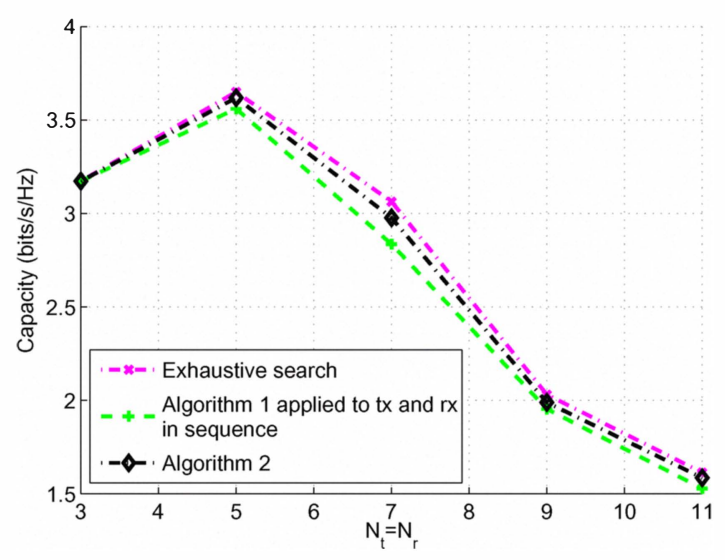

Fig. 3. Capacity with selection of both transmit and receive antennas is shown with $N_{t}$ or $N_{r}$ for $\rho=0 \mathrm{~dB}, L_{t}=L_{r}=3, L=0.5 \lambda, \epsilon_{1}=0.8$, and $\epsilon_{2}=0.3$.

\section{CONCLUSiOnS}

We proposed antenna selection algorithms for a single-user MIMO channel, which takes into account mutual coupling effect. The proposed schemes shown to perform close to an exhaustive search with much less complexity. Search complexity is a major issue since the selection of antennas happens in

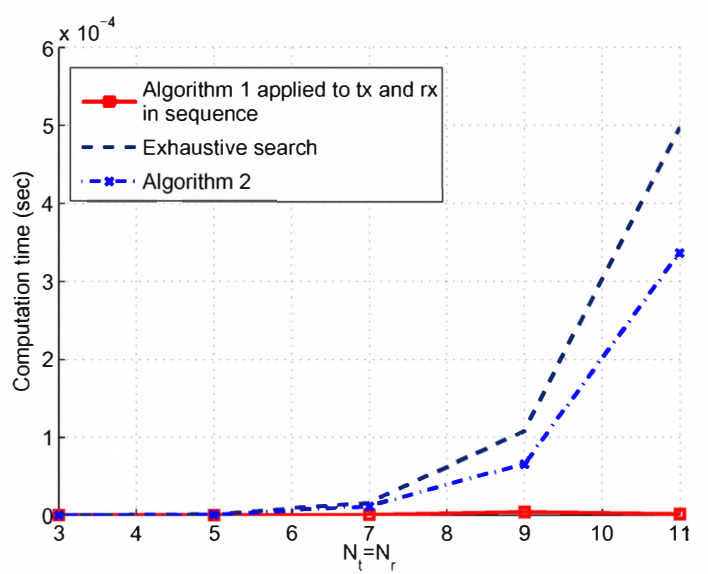

Fig. 4. Computation time of the schemes whose achievable rates are shown in Fig. 3 is plotted with the number of transmit and receive antennas.

real time and cannot be done offline. Our search complexity depends heavily on the threshold on mutual impedance, which is currently obtained from numerical simulations. Also, performance of the proposed schemes is shown via numerical results. Thus, our future work includes analysis of the performance, which should give us more insights, as well as investigation on the optimal threshold for a given setup.

\section{REFERENCES}

[1] İ. E. Telatar, "Capacity of multi-antenna Gaussian channels," European Trans. on Telecommun., vol. 10, pp. 585-595, Nov. 1999.

[2] T. Marzetta and B. Hochwald, "Capacity of a mobile multiple-antenna communication link in Rayleigh flat fading," IEEE Trans. Inf. Theory, vol. 45, no. 1, pp. 139 -157, Jan. 1999.

[3] S. Shen, M. McKay, and R. Murch, "MIMO systems with mutual coupling: How many antennas to pack into fixed-length arrays?" in Int Symp. on Inf. Theory and its Applications (ISITA), Oct. 2010, pp. 531 $-536$.

[4] J. Wallace and M. Jensen, "Mutual coupling in MIMO wireless systems: A rigorous network theory analysis," IEEE Trans. Wireless Commun., vol. 3, no. 4, pp. 1317 - 1325, Jul. 2004.

[5] M. Win and J. Winters, "Analysis of hybrid selection/maximal-ratio combining in Rayleigh fading," IEEE Trans. Commun., vol. 47, no. 12, pp. $1773-1776$, Dec. 1999.

[6] D. Gore, R. Nabar, and A. Paulraj, "Selecting an optimal set of transmit antennas for a low rank matrix channel," in IEEE Int. Conf. on Acoustics, Speech, and Signal Processing (ICASSP), vol. 5, 2000, pp. $2785-2788$ vol.5.

[7] S. Sandhu, R. Nabar, D. Gore, and A. Paulraj, "Near-optimal selection of transmit antennas for a MIMO channel based on shannon capacity," in 34th Asilomar Conf. on Signals, Systems and Computers, vol. 1, 2000, pp. $567-571$ vol.1.

[8] A. Gorokhov, D. Gore, and A. Paulraj, "Receive antenna selection for MIMO spatial multiplexing: Theory and algorithms," IEEE Trans. Signal Process., vol. 51, no. 11, pp. 2796 - 2807, Nov. 2003.

[9] A. Gorokhov, "Antenna selection algorithms for MEA transmission systems," in IEEE Int. Conf. on Acoustics, Speech, and Signal Processing (ICASSP), vol. 3, May 2002, pp. III-2857 -III-2860.

[10] L. Dai, S. Sfar, and K. Letaief, "Optimal antenna selection based on capacity maximization for MIMO systems in correlated channels," IEEE Trans. Commun., vol. 54, no. 3, pp. 563 - 573, Mar. 2006.

[11] W. C. Jakes, Microwave Mobile Communications. New York: Wiley, 1975 .

[12] C. A. Balanis, Antenna Theory: Analysis and Design. New York: Wiley, 1997.

[13] M. Gharavi-Alkhansari and A. B. Gershman, "Fast antenna subset selection in MIMO systems," IEEE Trans. Signal Process., vol. 52, no. 2, pp. 339-346, Feb. 2004. 